\title{
Metodologia de estimação da demanda por turismo doméstico no Brasil: aspectos teóricos e evidências empíricas ${ }^{1}$
}

José Roberto de Lima Andrade ${ }^{2}$

Resumo: O turismo vem se constituindo atualmente em uma das mais importantes atividades econômicas do mundo, englobando os diversos componentes e motivações da viagem. O processo de escolha do consumidor dos produtos turísticos vem sendo abordado na literatura sob os mais variados aspectos. $\mathrm{O}$ artigo enfatiza a abordagem econômica da demanda por turismo e sua evidência empírica ao mercado de turismo doméstico no Brasil.

Palavras-chave: Demanda por turismo; turismo doméstico no Brasil; modelo econométrico de demanda por turismo.

\section{Demand estimation for domestic tourism in Brazil: theoretical aspects and empirical evidence}

\begin{abstract}
Tourism is now one of the most important economic activities in the world. Behind it, there are several components and motivations for "a trip." The consumer's choice of tourism products has been approached in the literature from different perspectives. This article emphasizes the economic approach of tourism demand and the market evidence for domestic tourism in Brazil.
\end{abstract}

Key words: Tourism demand; domestic tourism in Brazil; econometric model.

JEL: $L 83$

\footnotetext{
1 Uma primeira versão deste artigo foi apresentada no V Encontro de Economistas de Língua Portuguesa. Recife, 2003.

2 Universidade Federal de Sergipe.
} 


\section{Introdução}

Entender o comportamento do consumidor é uma tarefa extremamente complexa e que requer a contribuição de diversos campos da ciência. De uma forma mais geral, o comportamento humano, segundo a abordagem desenvolvida por Maslow (1989) é motivado pela busca de satisfação de cinco grandes necessidades: fisiológicas, segurança, amor, estima e auto-realização.

Dentro do processo de tomada de decisões do consumidor por determinado produto ou serviço, podemos destacar o consumo de viagens de lazer, que deve ser aqui entendido como um conceito mais amplo que a simples motivação por descanso ou recreação, incluindo também os aspectos históricos e culturais. As viagens de lazer, inseridas na atividade turística, são uma das características marcantes da nossa sociedade.

Fundamentalmente, as viagens de lazer estão associadas ao turismo, atividade que tem como característica a própria viagem, entendida não apenas pelo deslocamento em si, mas por toda a estrutura que a viabiliza, ou seja, tanto atividades que precedem o deslocamento quanto as que possibilitam a permanência da pessoa que realizou o deslocamento no local de destino. Todavia, é importante destacar que, ao falarmos de turismo, estamos nos referindo não somente às viagens de lazer, mas aos diversos tipos de viagem, ou mais explicitamente, às diversas motivações da viagem.

O estudo do comportamento do consumidor é abordado na teoria econômica no tópico relacionado à teoria da demanda, que tradicionalmente aborda a demanda por lazer como uma questão derivada da oferta de trabalho. Mesmo para alguns autores que relacionam a economia ao turismo, como Eadington \& Redman (1991), Lundberg et al. (1995) Palomo (1990) e Sinclair \& Stabler (1997), a demanda por turismo em nada se diferencia, do ponto de vista conceitual, da abordagem tradicional da microeconomia.

A não consideração das especificidades do turismo e a adoção de modelos de demanda turística utilizando uma "teoria microeconômica geral" têm levado a resultados empíricos não satisfatórios, conforme poderá ser visto nos trabalhos realizados por Athyaman (1997) e Lim (1998).

O objetivo principal do artigo é apresentar um referencial teórico de análise econômica da demanda por turismo que possa embasar a realização de trabalhos empíricos, gerando resultados satisfatórios. Uma aplicação do referencial teórico proposto será feita através da utilização do modelo econométrico de demanda por turismo doméstico no Brasil. 
O artigo está dividido em quatro pontos. O primeiro aborda a importância da análise da demanda no entendimento do fenômeno turístico. O segundo demonstrará o referencial teórico proposto para a análise econômica da demanda por turismo. O terceiro ponto mostrará a utilização do referencial teórico na análise empírica da demanda por turismo doméstico no Brasil. Finalmente, o quarto ponto mostrará as considerações finais do artigo.

\section{A importância do consumidor na análise do turismo}

É possível encontrar inúmeras definições de turismo. Para a Organização Mundial de Turismo-OMT, órgão oficial do turismo mundial, o turismo é conceituado como "o conjunto de atividades que compreende a viagem de pessoas para locais fora da sua residência habitual, por menos de um ano, por lazer, trabalho, ou outros motivos" (OMT 1995:2).

Essa definição, de certa forma, dá a dimensão da atividade turística atual, algo que transcende o conceito clássico de viagem de lazer. Seguindo a definição da OMT (2000), é possível estabelecer, considerando como referência um determinado país (ou local), tipos de turismo. Desta forma, três tipos de turismo podem ser distinguidos:

1. Turismo doméstico é o turismo de residentes dentro do território do país (ou local) de referência;

2. Turismo interno é o turismo de não residentes dentro do território de referência, e;

3. Turismo externo é o turismo dos residentes fora do território de referência.

A definição de turismo da OMT privilegia, de certa forma, as atividades oriundas da viagem. Em outras palavras, pode-se afirmar que a ênfase é dada na relação entre cliente-consumo ocorrida durante o processo da viagem.

Uma definição de turismo que privilegie os aspectos motivacionais, ou de decisão da viagem, pode ser encontrada em Leiper (1993). O autor inicia a sua definição de turismo partindo da constatação que as diferentes definições existentes de turismo refletem, de certa forma, as diferentes perspectivas dos estudiosos e pesquisadores, que tendem a definir o turismo segundo sua própria disciplina ou perspectiva pessoal. Assim, é possível observar uma definição econômica, ambientalista, holística, etc. Essa opinião é compartilhada por Moore et al. (1995), que acreditam que as diversas conceitualizações de turismo refletem os diferentes interesses na análise do fenômeno turístico. 
Leiper (1993) considera que uma base acadêmica mais apropriada para a definição de um conceito de turismo provém do significado das terminologias "ismo" e "istas". Assim, “(...) Como o idealismo é a teoria e prática dos idealistas e o socialismo dos socialistas, também turismo é fundamentalmente relacionado aos turistas." (Leiper 1993:544).

Desta forma, seguindo a concepção de Leiper (1993), o turismo é antes de tudo uma forma de comportamento humano. Nessa perspectiva, o conceito de turismo pode ser definido como: "Turismo é um conjunto de idéias, de teorias ou ideologias advindas de pessoas na função turística, quando estas idéias são postas em prática" (Leiper 1993:544-5).

O conceito de turismo definido por Leiper (1993) é interessante à medida que transcende, no conceito de viagem, o aspecto do deslocamento, e insere, como ponto relevante, os aspectos comportamentais que levam a este deslocamento.

É importante ressaltar que, paradoxalmente, a definição de Leiper não está desprovida de uma "perspectiva própria”, ou seja, a sua crítica em relação à perspectiva pessoal que norteia as diversas definições de turismo é de certa forma repetida à medida que privilegia, no seu conceito de turismo, o aspecto motivacional da viagem. Assim, o conceito de Leiper não deve ser entendido enquanto uma perspectiva "ideal" de conceito de turismo, mas, um entendimento do fenômeno turístico que possa ter no componente motivacional da viagem um referencial de análise.

\section{Abordagem econômica da demanda por turismo: construção de um modelo de análise}

A literatura da análise econômica do turismo, apesar de numerosos estudos, concentra-se, basicamente, em três grandes áreas: análise da demanda, estimação do fluxo turístico e impacto econômico da atividade (Sinclair \& Stabler 1997). Em nível da teoria econômica, a discussão mais próxima da demanda por turismo aparece nos manuais de microeconomia como derivada da oferta de trabalho, como exposto, por exemplo, em Varian (1999). Para o autor, o lazer, entendido como o conjunto de atividades ligadas ao tempo livre, é considerado como um bem normal, ou seja, níveis maiores de renda resultam em níveis maiores de consumo de lazer.

Basicamente, a discussão lazer-trabalho exposta nos manuais de microeconomia tem como objetivo principal demonstrar a ambigüidade que existe em relação ao comportamento da oferta de trabalho. Essa ambigüidade está relacionada à variação nas taxas de salário. Níveis 
salariais maiores levariam a uma redução na quantidade ofertada de trabalho e, conseqüentemente, a um aumento na demanda por lazer.

Existem diversos autores que tentam relacionar a teoria econômica ao turismo. Dentre eles podemos destacar Cook (1994); Bull (1998); Eadington \& Redman (1991); Lundberg et al. (1995); Palomo (1990) e Sinclair \& Stabler (1997). Em relação especificamente à análise da demanda por turismo, a abordagem utilizada pela maioria destes autores é, em essência, semelhante à utilizada nos manuais de microeconomia, ou seja, tratam a demanda por turismo dentro do escopo geral da teoria da demanda, relacionado-a a fatores como preço, renda e preço de outros bens, além de incluir o aspecto relacionado à oferta de trabalho. Uma frase que representa esta abordagem pode ser observada em Eadington \& Redman (1991:45):

\begin{abstract}
Para o consumidor, o problema básico de escolha é formalizado em algumas decisões relacionadas. Um recurso que a maioria dos indivíduos ou consumidores controlam é o tempo. Assim, o problema inicial para o consumidor é encontrar uma alocação 'ótima' de tempo entre trabalho, lazer e o consumo de outros produtos. Trabalho, pensado inerentemente como menos desejável que lazer, será escolhido em relação ao lazer, desde que haja uma recompensa de renda em forma de salário.
\end{abstract}

De forma semelhante, segundo Sinclair e Stabler (1997), “(...) decisões de comprar turismo são tomadas em conjunto com as decisões de oferta de trabalho, também pode ser indicado que decisões de comprar turismo estão relacionadas às decisões de consumir outros bens e serviços (...)” (Sinclair \& Stabler 1997:33).

Para Palomo (1990), o turismo deixou de ter a característica de um bem de luxo, tornando-se um bem de consumo cada vez mais normal no cotidiano das pessoas, muito embora não se possa afirmar ainda que turismo possa ser considerado um bem necessário. Mesmo reconhecendo a importância dos diversos fatores que influenciam a demanda por turismo, o autor elabora uma função de demanda turística semelhante à função geral de demanda que é definida como:

$\mathrm{DT}_{\mathrm{t}}=\mathrm{f}\left(\mathrm{PT}_{\mathrm{t}}, \mathrm{Pi}_{\mathrm{t}}, \mathrm{Y}_{\mathrm{t}} \mathrm{TC}_{\mathrm{t}}, \mathrm{U}_{\mathrm{t}}\right)$, onde

$\mathrm{DT}_{\mathrm{t}=}$ Demanda Turística

$\mathrm{PT}_{\mathrm{t}=}$ Preços dos serviços turísticos

$\mathrm{Pi}_{\mathrm{t}=}$ Preços dos outros bens e serviços

$\mathrm{Y}_{\mathrm{t}=}$ Renda per capita 
$\mathrm{TC}_{\mathrm{t}=}$ Taxa de câmbio (quando o consumo turístico é efetuado no exterior), e;

$\mathrm{U}_{\mathrm{t}=}$ Variável estocástica

Athyaman (1997), ao realizar um levantamento de 20 modelos econométricos de demanda turística publicados em diversos artigos entre 1966 e 1990, constatou que, na sua totalidade, esses estudos se utilizaram da "teoria econômica básica da demanda" na determinação das suas variáveis explicativas. As variáveis explicativas utilizadas foram: preço do bem, preço dos bens substitutos, renda média, preferências, distribuição de renda e tamanho da população. Alguns estudos utilizaram outras variáveis explicativas, como custo de viagem, distância, atrações, climas, férias pagas, fatores demográficos, eventos especiais, custos de promoção e época de viagem.

O levantamento feito por Athyaman demonstrou que 70\% dos modelos possuíam variáveis que não tinham significância estatística ou apresentaram sinais dos coeficientes contrários à teoria econômica. Para o autor,

\begin{abstract}
Este estudo confirma a observação de Crouch de que alguns estudos de demanda por turismo têm utilizado variáveis que produzem um sinal inesperado na análise estatística. A afirmação de Crouch é de que as pesquisas em demanda por turismo são caracterizados por variáveis pobres (...) (Athyaman 1997:227):
\end{abstract}

Um outro estudo semelhante ao de Athyaman foi feito por Lim (1998), que realizou um levantamento de 100 artigos sobre modelos de demanda de turismo internacional publicados em diversas revistas nas últimas três décadas.

Para o autor,

(...) as variáveis proxy para destinações competitivas, gastos em marketing, viagens a negócios, e especialmente fatores qualitativos (...), refletem a insuficiente atenção que vem sendo dada em capturar os efeitos destas variáveis na demanda por turismo (...) (Lim 1998: $74)$.

Uma crítica mais contundente à utilização da teoria econômica da demanda na análise da demanda por turismo é feita por Morley (1992) e principalmente por Smeral (1989). Para esse autor, o equívoco da aplicação da teoria econômica ${ }^{3}$ na demanda por turismo reside principal-

3 A expressão teoria econômica da demanda utilizada no trabalho refere-se à abordagem neoclássica da demanda. 
mente no fato de que o objeto de consumo analisado na demanda por turismo não é considerado, ou seja, o produto turístico é percebido como uma commodity, um bem cujas características não são ressaltadas enquanto geradoras de utilidade para o consumidor.

Apesar das várias definições existentes sobre produto turístico, podemos utilizar o conceito desenvolvido por Acerenza (1991), que considera o produto turístico como um conjunto de prestações materiais e imateriais que são oferecidas com o propósito de satisfazer os desejos e expectativas dos turistas. $O$ produto turístico pode ser dividido em atrativos (elementos que determinam a escolha do turista), facilidades (permitem a permanência do turista no local visitado) e acesso (meios que possibilitam o deslocamento do turista).

Existem diversos autores que, dentro dos pressupostos gerais da teoria econômica neoclássica, consideram o objeto de consumo como uma cesta de atributos, característica essa do produto turístico. Os primeiros estudos teóricos nesse sentido remontam à década de 1960, com o trabalho Lancaster (1966). O trabalho de Rosen (1974) consolida as principais idéias do que podemos denominar de abordagem hedônica.

Utilizando-se do referencial teórico dos autores citados no parágrafo anterior, pode-se formular um referencial de análise econômica da demanda por turismo a partir dos seguintes pressupostos:

1. o turista age de maneira racional e procura maximizar a utilidade de sua viagem;

2. as restrições de consumo do turista são impostas pela sua restrição orçamentária e pelo tempo disponível para a realização da sua viagem, e

3. o turista maximiza a utilidade de sua compra em função do conjunto de características contidas no produto turístico.

Em relação ao primeiro aspecto, para a teoria microeconômica neoclássica, o processo de escolha do consumidor está baseado em duas premissas: racionalidade e maximização de utilidade. Os conceitos de racionalidade e de maximização da utilidade devem ser entendidos não numa perspectiva formal, ou seja, como um resultado final do comportamento do consumidor, mas em uma perspectiva, conforme exposto por Silberberg \& Suen (2001), da atitude do consumidor em buscar extrair o máximo do seu processo de compra.

O segundo ponto a considerar refere-se às restrições de compra do turista. Do ponto de vista estritamente econômico, a restrição de compra é dada pela limitação orçamentária do consumidor. Entretanto, ao considerarmos que a compra do turista (a viagem) implica deslocamento, um fator importante na restrição de compra é o tempo disponível para 
a realização deste deslocamento. O tempo, enquanto fator de restrição, é uma característica peculiar da demanda turística e deve ser inserido, conjuntamente com a restrição orçamentária, na definição das limitações de compra impostas ao turista na análise do seu processo de demanda.

Analisados o comportamento do consumidor e as suas restrições de consumo, cabe agora expor o terceiro ponto, que deve ser considerado como o mais importante, tanto em termos de adequação da teoria econômica à análise da demanda por turismo, quanto na aplicação de trabalhos empíricos. O ponto em questão refere-se ao objeto de consumo do turista, ou seja, ao produto turístico.

Desta forma, à medida que o produto turístico é um conjunto de características, o turista maximiza a utilidade de sua compra em função do conjunto destas características contidas no seu objeto demandado (produto turístico).

Suponha que as preferências de cada consumidor prestes a fazer uma viagem de turismo possam ser representadas por uma função de utilidades $U(\mathbf{z}, x)$ na qual $\mathbf{z}=Z_{-}^{-}, Z_{-}{ }_{2} \ldots Z_{-}{ }_{n}$ é o vetor que mede as quantidades ou intensidades dos $n$ atributos relevantes para as escolhas dos consumidores relativas a viagens de turismo e $x$ é o total de renda disponível para o consumo dos outros bens. Suporemos aqui que $U(\mathbf{z}, x)$ seja contínua, diferenciável, estritamente quase-côncava e que $\left(\mathbf{z}^{*}, x^{*}\right)>\left(\mathbf{z}^{\prime}, x^{\prime}\right) \Rightarrow U\left(\mathbf{z}^{*}, x^{*}\right)>U\left(\mathbf{z}^{\prime}, x^{\prime}\right)$. O custo monetário com a viagem de turismo depende das características dessa viagem e é dado pela função $p(\mathbf{z})$. Essa função costuma ser chamada de função de preços hedônicos. O problema de um consumidor com uma renda monetária $y$ é, nesse caso:

\section{Maximizar $U(\mathbf{z}, x)$}

Respeitando as condições: $x, \mathbf{z} \geq 0$ e $p(\mathbf{z})+\tilde{x} y \geq 0$.

As condições de máximo de primeira ordem, além dessas restrições, são sintetizadas, para o caso em que $z_{i}>0$, por

$$
\frac{U_{i}(\mathbf{z}, x)}{U_{1}(\mathbf{z}, x)}=p_{i}(\mathbf{z}) .4
$$

Notaremos por $\mathbf{z}^{*}=\left(z_{1}{ }^{*}, Z_{2}{ }^{*} \ldots z_{n}{ }^{*}\right)$ o vetor de atributos da viagem de turismo que resolve essa condição.

Essa condição pode ter uma interpretação econômica simples: a taxa marginal de substituição entre um atributo $i$ da viagem de turismo e a renda disponível para consumo de outros bens deve ser igual ao preço

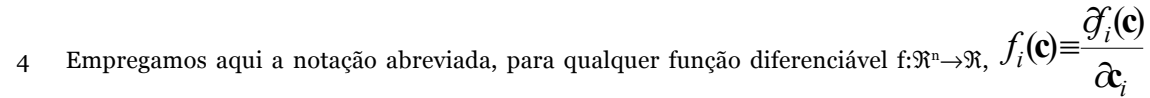


marginal de se aumentar a quantidade ou intensidade dessa característica nessa viagem.

Para uma visualização gráfica desse resultado, considere a função $\theta \mathbf{z}, y, u)$ definida por $U(\mathbf{z}, \tilde{y} \cdot(\mathbf{z}, y, u)) \equiv u$. Tal função define, dada $y$, hipersuperfícies de indiferença no espaço $\mathbf{z} \times p$ ou seja, combinações entre características da viagem de turismo e o preço dessa viagem que são indiferentes entre si. Na figura 2 a seguir são ilustradas curvas de nível dessa função para $\left(z_{2}, z_{3} \ldots z_{n}\right)=\left(z_{2}{ }^{*}, z_{3}{ }^{*} \ldots z_{n}{ }^{*}\right)$ projetadas no plano $z_{1} \times p$. Note que, uma vez que quanto menor o preço pago, maior é a utilidade do consumidor, quanto mais abaixo estiver a curva $\theta$ no gráfico, melhor estará o meu consumidor. Como conseqüência, $u^{1}<u^{*}<u^{0}$.

FIGURA 1 - EQUILÍBRIO DO CONSUMIDOR

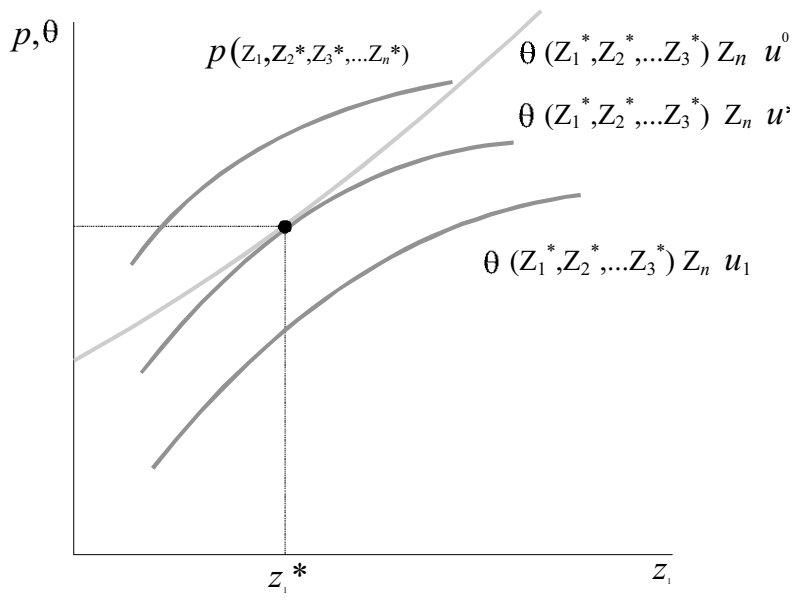

A curva $p\left(z_{1}, z_{2}^{*}, z_{3}^{*} \ldots z_{n}^{*}\right)$ representa a curva de nível também calculada para $\left(z_{2}, z_{3} \ldots z_{n}\right)=\left(z_{2}{ }^{*}, z_{3}{ }^{*} \ldots z_{n}{ }^{*}\right)$ da restrição orçamentária do consumidor. Este deverá procurar consumir sobre o ponto de sua restrição orçamentária que passe pela curva de indiferença mas que corresponda ao maior nível de utilidade possível. A solução desse problema se dá quando $z_{1}=z_{1}^{*}$, ou seja, no ponto de tangência entre a curva de restrição orçamentária e a curva de indiferença. A inclinação da linha de restrição orçamentária nesse ponto é dada por $p_{1}\left(\mathbf{z}^{*}\right)$ e a inclinação da curva de indiferença é dada por $\frac{U_{i}\left(\mathbf{z}^{*}, y-p_{i}\left(\mathbf{z}^{*}\right)\right)}{U_{1}\left(\mathbf{z}^{*}, y-p_{i}\left(\mathbf{z}^{*}\right)\right)}$.

A igualdade entre essas duas inclinações corresponde à condição (1). Evidentemente, consumidores diferentes possuem preferências diferentes. Isso deve fazer com que a cesta de atributos escolhida por um con- 
sumidor não seja necessariamente igual à escolhida por outro. A figura 2 ilustra essa situação supondo que o número de atributos seja igual a 1.

FIGURA 2 - EQUILÍBRIO PARA DOIS CONSUMIDORES DIFERENTES

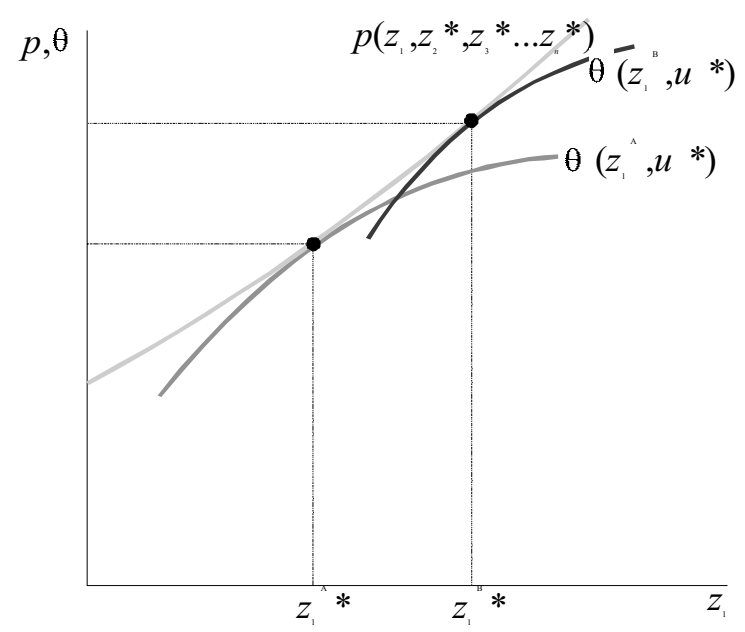

Este modelo de análise econômica da demanda por turismo é aplicável tanto para um único destino como para múltiplos destinos, à medida que estes também podem ser considerados como um produto turístico. É possível considerar que as características podem estar dispostas em mais de um destino, seguindo o conceito de atrações cumulativas de Lue (1993). O aspecto fundamental na análise é considerar que o turista procura maximizar um conjunto de características contidas no produto turístico, não importando se elas estão dispostas em um único destino ou em mais de um destino.

Obviamente que a consideração da seleção de multidestinos apresenta diversas vantagens, como a otimização do tempo, dos custos e da satisfação das diferentes motivações, considerando as viagens feitas em grupo. Todavia, mais uma vez, estas considerações podem ser incorporadas sem problema tanto nos pontos que compõem o referencial de análise proposto como no modelo de comportamento de turista.

\section{Demanda por turismo doméstico Brasil: características e evidências empíricas}

Os estudos relacionados a aspectos do turismo doméstico no Brasil são recentes, muito embora a EMBRATUR tenha realizado em 1974 uma pesquisa visando identificar as principais motivações do turista brasileiro. 
Pode-se afirmar que o primeiro grande estudo sobre o mercado doméstico de turismo no Brasil foi realizado em 1998 pela Embratur, em conjunto com a Fundação Instituto de Pesquisas Econômicas da Universidade de São Paulo (FIPE-USP), intitulada "Estudo, Caracterização e Dimensionamento do Mercado Doméstico de Turismo no Brasil" 5 . O estudo segmentou o mercado doméstico turístico em dois grandes grupos de renda. O primeiro grande grupo engloba faixas maiores de renda $(\mathrm{ABC})$, representando cerca de $40 \%$ da população brasileira, enquanto o outro grande grupo englobaria as faixas de renda menores (DE), representando os outros $60 \%$ da população.

Considerando apenas os gastos realizados pelos turistas domésticos, a importância econômica do turismo doméstico no Brasil pode ser observada no quadro 1 a seguir:

QUADRO 1 - IMPACTOS ECONÔMICOS DO TURISMO DOMÉSTICO NO BRASIL

\begin{tabular}{|c|c|}
\hline $\begin{array}{c}\text { Participação no PIB (relativo a } \\
\text { 2001) }\end{array}$ & $\begin{array}{c}\text { Consumo doméstico (gastos } \\
\text { turísticos) }\end{array}$ \\
\hline $4,1 \%$ & $\mathrm{R} \$ 48,4$ bilhões \\
\hline
\end{tabular}

FONTE: FIPE/EMBRATUR-2002.

O tamanho do mercado do turismo doméstico no Brasil é estimado em 41.352.00o turistas, o que corresponde a aproximadamente $24 \%$ da população brasileira (dados de 2002).

Apesar de representar apenas 4,13\% da população, as famílias com classe de renda com mais de 15 salários mínimos respondem por $54,8 \%$ do total de turistas no Brasil, o que demonstra o baixo acesso que a maioria da população brasileira tem às viagens.

Uma outra característica marcante do turismo doméstico no Brasil é o perfil intra-regional das viagens. As regiões (e estados) com maior contingente populacional são, na maioria dos casos, os maiores emissores e receptores do fluxo turístico doméstico.

A principal motivação das viagens domésticas do turismo doméstico é o lazer, com 76,1\% (média Brasil). Entretanto, uma análise superficial poderia induzir a uma caracterização de um perfil de turista bastante semelhante ao "turista padrão" dos livros texto. Desagregando as motivações das viagens por lazer, percebemos que aproximadamente $74 \%$

5 A pesquisa foi realizada no início de 2006. Como os resultados ainda são preliminares, optou-se por utilizar os dados consolidados da pesquisa de 2002. 
ANDRADE, J. R. L. Metodologia de estimação da demanda...

correspondem a visitas a amigos e parentes, conforme pode ser observado no quadro 2.

QUADRO 2 - PRINCIPAIS MOTIVAÇÕES DAS VIAGENS DOMÉSTICAS NO BRASIL

\begin{tabular}{|c|c|}
\hline Tipo de viagem & Brasil \\
\hline Lazer & $76,1 \%$ \\
Não lazer & $23,9 \%$ \\
\hline \multicolumn{2}{|c|}{ Razões das viagens de lazer } \\
\hline Visitar amigos / parentes & $50,2 \%$ \\
Fuga da rotina / descanso & $27,5 \%$ \\
Atrativos Turísticos & $11,5 \%$ \\
Veranismo & $4,3 \%$ \\
Atrativos Culturais & $2,5 \%$ \\
Estância Climática & $0,9 \%$ \\
Prática esporte por lazer & $0,8 \%$ \\
Compras por lazer & $0,7 \%$ \\
Ecoturismo & $0,4 \%$ \\
Parques temáticos & $0,3 \%$ \\
Assistir comp. Esportivas & $0,2 \%$ \\
Resorts, Hotéis, ou Spas & $0,1 \%$ \\
Outras & $0,5 \%$ \\
\hline
\end{tabular}

FONTE: FIPE/EMBRATUR-2002.

A utilização de modelos econométricos na análise da demanda por turismo tem sido feita desde os anos 50 do século passado. No Brasil, a utilização de métodos econométricos na análise da demanda por turismo é ainda incipiente, destacando-se os trabalhos de Lage (1988), que tenta estimar a demanda por passagens aéreas no Brasil, e Rabahy (1990), que elaborou um modelo para estimar o fluxo internacional de turismo para o Brasil. Mais recentemente, Cruz (2004) elaborou modelo em que analisa os determinantes do turismo internacional utilizando metodologia de estimação de dados em painel. Em relação à análise da demanda doméstica de turismo no Brasil, não se observa até o momento a existência de nenhum estudo que utilize métodos econométricos que considere, especificamente, os fatores gerais na determinação das viagens domésticas. Os dados utilizados no modelo são referentes às trinta maiores destinações turísticas (cidades receptoras) da pesquisa Embratur/Fipe (1998).

O modelo de demanda por turismo doméstico no Brasil é formalmente especificado como: 
Flt $=\beta_{1}+\beta_{2}$ ATRAT $+\beta_{3}$ DISTPOND $+\beta_{4}$ REND $+\mu_{i}$, onde:

Flt $=$ Fluxo de turistas

ATR $=$ número de atrações

DISTPOND = Distância ponderada dos principais centros emissores

REND = Renda média per capta dos principais centros emissores

$\beta_{1}=$ intercepto (constante) do modelo

$\beta_{2}, \beta_{3}, \beta_{4}=$ parâmetros do modelo

$\mu_{i}=$ erro (perturbação estocástica)

A variável explicativa número de atrações (ATRAT) procura captar, conforme descrito no referencial de análise econômica da demanda por turismo, a consideração de que o produto turístico é um conjunto de atributos, e que os consumidores avaliam este produto em função da quantidade de atributos que ele contém. Entretanto, pelo próprio conceito de produto turístico, estes atributos refletem não só características tangíveis, mas também intangíveis, como por exemplo, a atratividade proporcionada pelas manifestações culturais. Assim, na ausência de dados que representem com fidelidade as características do produto turístico, a variável explicativa número de atrações deve ser considerada como uma proxy do número de atributos do produto turístico.

Foram considerados os atrativos turísticos das cidades selecionadas através da classificação proposta pelo Guia Quatro Rodas Brasil 2000. O guia classifica as atrações entre 1 e 4 estrelas, significando, respectivamente: de algum interesse, interessante, muito interessante e não deixe de ir. Como forma de qualificar melhor o nível das atrações das cidades, ponderou-se cada classificação em função do seu número de estrelas.

A variável distância está presente em todos os modelos de demanda por viagem observados na literatura sobre demanda por turismo, sendo considerado no modelo como uma proxy do custo de viagem. Como tentativa de representar da melhor forma a importância da distância, e tendo em vista o perfil intra-regional das viagens no Brasil, atribuiu-se um peso maior à distância do centro emissor, localizado na região diferente da do destino. Como o Estado de São Paulo é o principal emissor para as demais regiões do Brasil, atribuiu-se peso maior à distância do Estado de São Paulo (medida pela distância da capital) aos destinos localizados fora da Região Sudeste.

A atribuição de um peso que represente a importância da distância na determinação das viagens não é uma tarefa simples, na medida em que a 
atribuição deste peso, de certa forma, representa um "coeficiente de atrito" entre dois locais, na medida em que a distância não representa apenas o custo de viagem, mas um conjunto maior de atributos que pode refletir o nível de segurança (grau de conhecimento), interação econômica, etc. Esta dificuldade foi encontrada por Rabahy (1980) na determinação do modelo gravitacional de determinação da potencialidade das localidades turísticas, onde os valores do coeficiente de atrito dos modelos gravitacionais propostos na literatura variam, conforme o referencial adotado, de o,689 a próximo de 3.

Foram dados três valores $(1,5,2$ e 2,5) para o peso atribuído à distância da cidade de São Paulo aos destinos localizados fora da Região Sudeste. Os resultados dos modelos com as variáveis explicativas (distâncias ponderadas com os pesos 1,5, 2 e 2,5) não demonstraram diferenças significativas. Entretanto, optou-se por considerar o peso 2 (DISTPOND) como o melhor parâmetro atribuído, em função do resultado dos testes de significância do parâmetro.

Finalmente, foi utilizada a variável renda, que é um componente elementar de qualquer modelo de demanda. Os valores utilizados para o nível de renda foram obtidos a partir do nível de renda per capita (dados de 1997) dos estados fornecidos pelo IBGE. Considerou-se a renda média dos principais estados emissores.

A não disponibilidade de uma série histórica significativa para todos os dados fez com que se utilizassem as informações referentes a um único período (cross-section).

Os resultados mais relevantes das equações dos modelos estimados estão descritos na Tabela 1, a seguir: 
TABELA 1 - RESULTADOS DO MODELO ECONOMÉTRICO DE DEMANDA POR TURISMO DOMÉSTICO NO BRASIL

\begin{tabular}{|c|c|c|c|c|}
\hline \multirow[b]{2}{*}{ Equação 1} & \multicolumn{4}{|c|}{ Valores dos Coeficientes ( $\beta$ 's) das Variáveis* } \\
\hline & Constante & ATRAT & DISTPOND & RENDA \\
\hline \multirow[t]{2}{*}{$\left(\mathrm{R}^{2}=0,635\right)$} & 17,246 & 1,349 & $-0,0083$ & $(0,0055)$ \\
\hline & $(0,279)$ & $-6,112$ & $(-0,561)$ & $(0,631)$ \\
\hline Equação 2 & Constante & ATRAT & DISTPOND & ** \\
\hline \multirow[t]{2}{*}{$\left(R^{2}=0,628\right)$} & 61,007 & 12,826 & $-0,0172$ & \\
\hline & $-4,053$ & $-6,436$ & $(-1,99)$ & \\
\hline Equação 3 & Constante & ATRAT & $* *$ & RENDA \\
\hline \multirow[t]{2}{*}{$\left(\mathrm{R}^{2}=0,631\right)$} & $-13,46$ & 1,396 & & 0,008 \\
\hline & $(0,477)$ & $-6,782$ & & $-2,053$ \\
\hline $\begin{array}{l}\text { Equação } 4 \text { (log- } \\
\text { linear) }\end{array}$ & Constante & ATRAT & DISTPOND & ** \\
\hline \multirow[t]{2}{*}{$\left(\mathrm{R}^{2}=0,524\right)$} & 44,514 & 0,4383 & $-0,2348$ & \\
\hline & $-67,929$ & $-49,723$ & $(-2,599)$ & \\
\hline $\begin{array}{l}\text { Equação } 5 \text { (log- } \\
\text { linear) }\end{array}$ & Constante & ATRAT & DISTPOND & RENDA \\
\hline \multirow[t]{2}{*}{$\left(\mathrm{R}^{2}=0,525\right)$} & $-29,617$ & 0,4975 & & 0,660 \\
\hline & $(-1,444)$ & $-5,544$ & $* *$ & $-2,897$ \\
\hline
\end{tabular}

*Valores dos testes $t$ estão em parênteses.

**Variável excluída do modelo.

Os resultados apresentados na Tabela 1 demonstram que a variável explicativa atrações (ATRAT) apresenta significância estatística, ou seja, possui poder explicativo sobre a demanda por turismo doméstico no Brasil em todas as regressões estimadas, confirmando um dos pontos do referencial de análise econômica da demanda por turismo proposto no trabalho. Em relação à não significância estatística das variáveis renda $\mathrm{e}$ distância ponderada (DISTPOND), quando expostas conjuntamente, demonstram uma clara presença de multicolinearidade.

Segundo Greene (1997), a multicolinearidade ocorre quando se observa a presença de correlação entre as variáveis, ou seja, é possível expressá-las como uma combinação linear umas das outras. A principal conseqüência da multicolinearidade é a impossibilidade de se estimar com precisão os coeficientes (parâmetros) das variáveis da regressão. O indício mais claro da sua presença pode ser observado quando, no resultado da regressão, o valor do $\mathrm{R}^{2}$ é alto, mas os valores das variáveis explicativas, quando estimados conjuntamente, não apresentam (em algumas das variáveis) significância estatística. Para Greene (1997), a solução mais trivial para a correção da multicolinearidade é a eliminação $\mathrm{da}(\mathrm{s})$ variável(is) que provavelmente estariam correlacionadas. 
Em relação ao modelo de demanda por turismo doméstico no Brasil, a multicolinearidade entre as variáveis explicativas distância ponderada e renda pode ser explicada em virtude do fato de que a natureza intraregional das viagens domésticas no Brasil representa o deslocamento de pessoas com níveis de renda, determinados regionalmente, semelhantes. Desta forma, a distância estaria refletindo, em parte, também o nível de renda de grande parte do fluxo turístico do destino.

Em relação aos valores esperados dos parâmetros das variáveis explicativas, os resultados dos modelos mais representativos (equações 2, 3 e 5) são os esperados pela teoria. Assim, observa-se uma relação positiva entre o número de atrações e a demanda por turismo, bem como do nível de renda. Em relação à variável explicativa distância ponderada, que reflete em parte o custo da viagem, o valor negativo do parâmetro é coerente com a teoria econômica da demanda.

Os modelos melhores ajustados foram aqueles especificados na forma linear (equações 2 e 3). Considerando os valores da equação 2, os resultados dos coeficientes podem ser interpretados de forma a proporcionar uma análise das implicações do resultado do modelo para a análise da demanda por turismo doméstico no Brasil. Do ponto de vista da variável explicativa atrações (ATRAT), o valor encontrado demonstra, de forma empírica, a importância dada à caracterização do produto turístico na análise econômica da demanda por turismo.

O valor do parâmetro da variável distância ponderada (DISTPOND) deve ser interpretado com bastante cuidado, na medida em que esta variável pode traduzir não só o custo da viagem, medido pela distância, como também o próprio nível de renda do turista, na medida em que ela pode captar esta característica em virtude do próprio perfil intra-regional do turismo no Brasil. Considerando apenas o aspecto do custo da viagem que a variável tenta captar, o valor encontrado (ver equação 2) é coerente com o proposto pela teoria econômica, na medida em que demonstra uma relação inversa entre o aumento da distância e a demanda por viagem doméstica no Brasil.

O valor da variável explicativa renda (equação 3) é também coerente com o proposto pela teoria econômica, já que demonstra uma relação positiva entre o nível de renda e a demanda por turismo. Uma consideração merece ser feita em relação ao valor do coeficiente encontrado $(0,008)$. Este pode ser interpretado como refletindo o nível de renda necessário para os gastos em viagens domésticas. Assim, a demanda por viagens domésticas só ocorreria, no caso brasileiro, a partir de níveis maiores de renda. Essa afirmativa é reforçada pela análise, no modelo 3 , do resultado do termo intercepto (C) do modelo. A sua não significância estatística (que também existe no modelo 5) permite afir- 
mar que não existe uma demanda "autônoma" de viagens domésticas no Brasil, ou seja, independente do nível de renda.

Ao se analisar o modelo especificado na forma log-linear (equação 5), a interpretação da variável explicativa renda é semelhante à do modelo especificada linearmente. $\mathrm{O}$ valor do coeficiente da variável explicativa renda (o,0001) deve ser interpretado como a elasticidade-renda da demanda por viagem doméstica no Brasil. A sua elevada inelasticidade reflete a relação existente entre níveis maiores de renda e a demanda por viagens domésticas no Brasil.

Dentre os cinco modelos apresentados, os modelos 2 a 5 podem ser considerados como representativos para a análise da demanda por turismo doméstico no Brasil, em função dos seguintes motivos:

Os modelos possuem variáveis explicativas coerentes com o referencial de análise econômica da demanda por turismo proposto no item anterior;

Os resultados dos parâmetros das variáveis explicativas são iguais aos esperados pelo referencial de análise econômica da demanda por turismo proposto e refletem, no caso da variável renda, o resultado obtido pela pesquisa Embratur/Fipe (1998);

Os modelos possuem um poder explicativo razoável, com estas variáveis explicando cerca de $63 \%$ da demanda por turismo doméstico no Brasil, no caso dos modelos especificados linearmente.

Apesar dos resultados obtidos, existe um conjunto de aperfeiçoamentos possíveis de serem feitos, e que estão relacionados principalmente a uma melhor qualificação das variáveis explicativas ${ }^{6}$. No caso brasileiro, observa-se ainda a inexistência de uma série histórica que permita a realização de trabalhos mais consistentes, com a utilização inclusive de outros métodos de estimação.

\section{Considerações finais}

O estudo da demanda por turismo é uma tarefa complexa, que requer tanto um aprofundamento teórico como comprovações empíricas. Os modelos e teorias apresentados neste trabalho constituem uma pequena parte da discussão, que certamente não se encontra ainda resolvida.

Todavia, a apresentação de referencial de análise econômica coerente com as especificidades da demanda por turismo pode contribuir não só para a realização de trabalhos empíricos, mas também com a tentativa

6 O que é um problema geral dos trabalhos econômicos sobre demanda por turismo, como pode ser observado em Athyaman (1997) e Lim (1998). 
de construção de uma área específica da economia dedicada à análise do turismo, o que certamente se justifica pela dimensão econômica que a atividade desempenha atualmente.

Em nível de Brasil, os resultados apresentados pela pesquisa Fipe/ Embratur $(1998,2002)$ são certamente um instrumento bastante importante para o entendimento da atividade turística no país. Entender a demanda por turismo é fundamental num país com produtos turísticos tão diversificados e condição primária tanto para a eficiência de políticas públicas no setor, quanto para o sucesso de empreendimentos privados.

O modelo econométrico de demanda por turismo doméstico no Brasil, apesar dos resultados satisfatórios apresentados, considerando que se trata de um trabalho pioneiro, pode ser aprimorado na medida em que se façam alguns ajustes, principalmente relacionados à coleta dos dados das variáveis explicativas propostas.

\section{Referências}

ANDRADE, J. R. L. (2002). Uma contribuição à análise econômica da demanda por turismo. São Paulo: Tese de Doutorado, Universidade de São paulo.

ACERENZA, Miguel. (1991). Promoção Turística: um enfoque metodológico. São Paulo: Pioneira.

ATHYAMAN, Adee. (1997). "Knowledge development in tourism: tourism demand research." Tourism Management 18(4): 221-228.

BULL, Adrian. (1998). La economía del sector turístico. Madrid: Alianza Editorial.

COOKE, Andrew. (1994). The Economics of leisure and sport. Great Britain: Routledge.

CROMPTON, John L. \& ANKOMAH, Paul K. (1993). "Choice set propositions in destination decisions." Annals of Tourism Research 20: 461-476.

CRUZ, M. J. V. (2004). Os determinantes do turismo internacional e as restrições à inserção dos países em desenvolvimento: Uma análise comparativa entre as regiões da América do Sul, África e Sul da Ásia. Curitiba: Dissertação de Mestrado, Universidade Federal do Paraná.

DELLAERT, Benedict G. C. \& ETTEMA, Dick F. \& LINDH, Crister. (1998). "Multi-faceted tourist travel decisions: a framework to describe tourists' sequential choices of travel components." Tourism Management 19(4): 313-320.

EADINGTON, William R. \& REDMAN, Milton. (1991). "Economics and Tourism." Annals of Tourism Research 18: 41-56.

EMBRATUR/FIPE. (1998 e 2002). Estudo, caracterização e dimensionamento do mercado doméstico de turismo no Brasil. São Paulo. 
GREENE, William H. (1997). Econometric Analysis. Prentice Hall.

GUIA QUATRO RODAS. (2000). Brasil.

LAGE, Beatriz H, G. (1988) Economia do Turismo: uma análise de suas influências sobre o comportamento dos consumidores. 1988. São Paulo: Tese de Doutorado, ECA/USP.

LANCASTER, Kelvin J. (1966). "A New Approach to Consumer Theory.” The Journal of Political Economy 74(2). (1991). Modern Consumer Theory. Edwarr Elgar Publishing.

LEIPER, Neil. (1993). "Defining Tourism and related concepts: tourist, market, industry, and tourism system." In KHAN, Mahmood \& OLSEN, Michael Var Turgut (Ed.) VNR's Encyclopedia of Hospitality and Tourism. New York.

LIM, Christine. (1998). "An econometric classification and review of international tourism demand models." Tourism Economics 3(1): 69-82.

LUE, C. (1993). "Conceptualization of multi-destination pleasure trips." Annals of Tourism Research 20: 289-301.

LUNDBERG, Donald E. \& STAVENGA, Mink H. \& KRISHNAMOORT, M. (1995). Tourism Economics. John Wiley \& Sons.

MASLOW, A. H. (1989). “A Theory of Human Motivation.” In LYNE, C. (Ed.). Leisure travel and tourism. Massachusetts: Institute of Certified Travel Agents.

MOORE, K. \& CUSHMAN, G. \& SIMMONS, D. (1995). "Behavioral conceptualization of tourism and leisure." Annals of Tourism Research 22(1): 67-85.

MORLEY, Clive L. (1998). "A dynamic international demand model." Annals of Tourism Research 25(1): 70-84.

. (1992). "A microeconomic theory of international tourism demand." Annals of Tourism Research 19: 250-267.

. (1994). "Experimental destination choice analysis." Annals of Tourism Research 21: 780-791.

OMT. (1995). Concepts, definitions and classifications of tourism statistics. Madrid.

PALOMO, Manuel Figuerola. (1990). Teoría económica del turismo. Madrid: Alianza Editorial.

RABAHY, Wilson Abrahão. (1990). Planejamento do turismo: estudos econômicos e fundamentos econométricos. São Paulo: Loyola.

(1980). Subsídios para uma política de desenvolvimento das atividades turísticas: Modelos Potencial e de Desempenho Real. São Paulo: Dissertação de Mestrado, Escola de Comunicações e Artes da Universidade de São Paulo.

ROSEN, S. (1974). "Hedonic prices and implicit markets: product differentiation in pure competition." The Journal of Political Economy 82: 34-35. 
ANDRADE, J. R. L. Metodologia de estimação da demanda...

RUGG, Donald. (1973). "The choice of journey destination: a theoretical and empirical analysis." The Review of Economics and Statistics 55(1): 64-72.

SILBERBERG, E. \& SUEN, W. (2001). The structure of economics: a mathematical analysis. McGraw-Hill.

SINCLAIR, M. T. \& STABLER, Mike. (1997). The economics of tourism. Routledge.

SMERAL, Egon. (1989). "Economic models of tourism." In WITT, Stephen F. \& MOUTINHO, Luiz. Tourism marketing and management handbook. Prentice Hall.

SMITH, Stephen. (1994). "The tourism product." Annals of Tourism Research 21(3): $582-595$.

SWARBROOKE, J. \& HORNER, S. (1999). Consumer behavior in tourism. Butterworth-Heinemann.

UM, S. \& CROMPTON, J. L. (1990). "Attitude determinants in tourism destination choice." Annals of Tourism Research 17: 432-448.

UM, S. "Pleasure travel destination choice." (1993). VNR's Encyclopedia of Hospitality and Tourism. New York.

URRY, John. (1996). O olhar do turista: lazer e viagem nas sociedades contemporâneas. São Paulo: Studio Nobel.

UYSAL, M. \& HAGAN, A. L. (1993). "Motivation of pleasure travel and tourism." VNR's Encyclopedia of Hospitality and Tourism. New York.

VARIAN, Hall, R. (1999). Intermediate microecnomics: a modern approach. Norton \& Company.

WITT, Christine A. \& WRIGHT, Peter L. (1994). "Tourism motivation: life after Maslow." In JOHNSON, Peter; THOMAS, Barry (Ed.). Choice and demand in tourism. Gret Bretain: Biddles.

WITT, Stephen F. \& MOUTINHO, Luiz. (1989). Tourism marketing and management handbook. Prentice Hall.

WORLD TOURISM ORGANIZATION BUSINESS COUNCIL. (2000). Changes in leisure time: the impact on tourism. Madrid: WTOBC.

Recebido em: 16 jun. 2006 Aceite em: 12 ago. 2006 